\title{
Comparison of measures of comorbidity for predicting disability 12-months post-injury
}

Belinda J Gabbe ${ }^{1,2,4^{*}}$, James E Harrison ${ }^{3}$, Ronan A Lyons ${ }^{4}$, Elton R Edwards ${ }^{1,5,6}$ and Peter A Cameron ${ }^{1,2,6}$

On behalf of the Victorian Orthopaedic Trauma Outcomes Registry

\begin{abstract}
Background: Understanding the factors that impact on disability is necessary to inform trauma care and enable adequate risk adjustment for benchmarking and monitoring. A key consideration is how to adjust for pre-existing conditions when assessing injury outcomes, and whether the inclusion of comorbidity is needed in addition to adjustment for age. This study compared different approaches to modelling the impact of comorbidity, collected as part of the routine hospital episode data, on disability outcomes following orthopaedic injury.

Methods: 12-month Glasgow Outcome Scale - Extended (GOS-E) outcomes for 13,519 survivors to discharge were drawn from the Victorian Orthopaedic Trauma Outcomes Registry, a prospective cohort study of admitted orthopaedic injury patients. ICD-10-AM comorbidity codes were mapped to four comorbidity indices. Cases with a GOS-E score of 7-8 were considered "recovered". A split dataset approach was used with cases randomly assigned to development or test datasets. Logistic regression models were fitted with "recovery" as the outcome and the performance of the models based on each comorbidity index (adjusted for injury and age) measured using calibration (Hosmer-Lemshow (H-L) statistics and calibration curves) and discrimination (Area under the Receiver Operating Characteristic (AUC)) statistics.
\end{abstract}

Results: All comorbidity indices improved model fit over models with age and injuries sustained alone. None of the models demonstrated acceptable model calibration ( $\mathrm{H}-\mathrm{L}$ statistic $\mathrm{p}<0.05$ for all models). There was little difference between the discrimination of the indices for predicting recovery: Charlson Comorbidity Index (AUC 0.70, 95\% Cl: 0.68, 0.71); number of ICD-10 chapters represented (AUC 0.70, 95\% Cl: 0.69, 0.72); number of six frequent chronic conditions represented (AUC 0.70, 95\% Cl: 0.69, 0.71); and the Functional Comorbidity Index (AUC 0.69, 95\% Cl: 0.68, 0.71).

Conclusions: The presence of ICD-10 recorded comorbid conditions is an important predictor of long term functional outcome following orthopaedic injury and adjustment for comorbidity is indicated when assessing risk-adjusted functional outcomes over time or across jurisdictions.

Keywords: Orthopaedic injury, Comorbidity, Disability outcomes, Prediction

\section{Background}

Measuring disability following injury is important. Understanding the factors that impact on disability is necessary to inform trauma care, and enable adequate risk-adjustment for benchmarking and monitoring. The presence of comorbidities has been identified as an important predictor of mortality following injury [1-3].

\footnotetext{
* Correspondence: belinda.gabbe@monash.edu

'Department of Epidemiology and Preventive Medicine, Monash University, The Alfred Centre, 99 Commercial Rd, Melbourne, Victoria 3004, Australia

${ }^{2}$ National Trauma Research Institute, The Alfred Hospital, Melbourne, Australia Full list of author information is available at the end of the article
}

Others have found that age is a more important predictor, and comorbidity offers little prediction benefit compared to models using age alone [4-6]. The impact of comorbidity on the prediction of disability outcomes has not been well explored. Untangling the effect of comorbidity has been identified as an important challenge for measuring injury-related disability $[7,8]$.

De Groot et al., identified numerous methods for measuring comorbidity, and highlighted many issues with the development and validation of comorbidity indices [9]. Of note were the lack of a consistent definition

\section{Biomed Central}


of comorbidity, variation in populations used to develop comorbidity measures, limited validation undertaken, and few measures developed with disability in mind [9]. Several studies have reported associations between comorbidity and disability [7,10-16] but none have directly compared indices.

This study compared different approaches to modelling the impact of comorbidity, collected as part of the routine hospital discharge data, on 12-month disability outcomes in an orthopaedic injury population to inform the development of risk adjustment models for disability outcomes. A key additional aim was to establish whether the inclusion of comorbidity provides additional important predictive value in addition to adjustment for age alone.

\section{Methods}

Data

The Victorian Orthopaedic Trauma Outcomes Registry (VOTOR) monitors the management and outcomes following admission to hospital for adults with acute orthopaedic injury [17]. This sentinel site registry collects data about all orthopaedic trauma admissions to four participating hospitals in the state of Victoria, Australia. The contributing hospitals include two adult major trauma services (Level 1 trauma centre equivalent), one large regional (rural) hospital, and one large metropolitan hospital, to ensure a wide representation of orthopaedic trauma patients in the registry. All eligible cases are included on the registry using an opt-off consent process [18]. The registry is approved by the Human Research Ethics Committee at each participating hospital and Monash University.

\section{Procedures}

Cases included survivors to hospital discharge, admitted between March 2007 and July 2010. Demographic, injury event, International Classification of Diseases 10th revision Australian Modification (ICD-10-AM) diagnosis codes (up to 40 per admission), and 12-month functional outcomes data were extracted for analysis.

ICD-10-AM orthopaedic injury diagnosis codes were mapped to 10 orthopaedic injury groups. Indicator variables were generated to represent important nonorthopaedic injuries, including variables for intracranial injury and/or skull fracture, multiple rib fractures, intraabdominal or intra-thoracic organ injury, and burns.

\section{Comorbidity measures}

ICD-10-AM diagnosis codes are the source of comorbidities for VOTOR. In Victoria, each code contains a prefix with "P" representing principal diagnosis requiring treatment during the stay, " $\mathrm{A}$ " representing additional diagnoses and " $\mathrm{C}$ " representing in-hospital complications.
Australian Coding Standards specify that most conditions should be coded as additional diagnoses if they affect treatment, require investigation, or use resources during the admission. However, the standards require coding of certain conditions whenever they are present; some communicable diseases (e.g. HIV, viral hepatitis), diabetes and pregnancy [19].

For the purposes of this study, the following were excluded from mapping to comorbidity measures:

i. All in-hospital complications ("C" prefix codes);

ii. Chapter XIX (Injury, poisoning and certain other consequences of external causes) with a "P" prefix (indicating an injury principal diagnosis);

iii. All Chapter XVIII (Symptoms, signs and abnormal clinical and laboratory findings, not elsewhere classified) as these are not comorbidities;

iv. Chapters XX (External causes of morbidity and mortality) to XXII (Codes for special purposes) as these are not comorbidities.

Remaining "P" and "A" prefixed codes were checked by the authors (PAC, RAL, BJG) to remove erroneously coded complications. These included acute posthaemmorhagic anaemia, acute subendocardial myocardial infarction, acute renal failure, acute respiratory infections, fever and post-traumatic amnesia. Codes remaining were assumed to represent comorbidities.

Four methods for classifying comorbidity were used and the ICD-10-AM codes mapped to the four indices. These were: (i) Charlson Comorbidity Index (CCI); (ii) Functional Comorbidity Index (FCI); (iii) categorisation by ICD-10 chapter; and (iv) the six conditions described by Haagsma et al. [7].

\section{Charlson comorbidity index (CCI)}

The 19 Charlson conditions were mapped to the CCI from the ICD-10-AM codes (Table 1), resulting in a weight of $1,2,3$ or 6 in accordance with Charlson's recommendations, and zero if no CCI codes were recorded [5]. The CCI is used widely for outcome adjustment in the injury literature $[10,11,14]$.

\section{Functional comorbidity index (FCI)}

The FCI was developed as a comorbidity index with physical function as the outcome of interest, using an 18-item (comorbidities) self-administered questionnaire where the FCI score is the sum of the number of conditions reported for the person (0-18) [20]. ICD-10-AM codes were mapped to the indicator variables (yes/no) for each FCI condition (Table 1) and FCI scores calculated as the sum of the conditions represented. 
Table 1 ICD-10 to comorbidity indices map

\section{Comorbidity Measure}

Functional Comorbidity Index

Haagsma et al. (2011)

Charlson Comorbidity Index

Arthritis
Osteoporosis
Asthma
COPD/ARDS
Angina
CHF or Heart disease
Heart Attack
Neurological disease
Stroke or TIA
Diabetes
PVD
Upper GI disease
Depression
Anxiety or panic disorders
Visual impairment
Hearing impairment
Degenerative disc disease
Obesity
Chronic non-specific lung disease
Heart disease
Diabetes
Backache
OA
Other disease or injury
RA

Myocardial infarction

Congestive heart failure

Peripheral vascular disease

Dementia

Cerebrovascular disease

Chronic pulmonary disease

Connective tissue disease

Ulcer disease

Mild liver disease

Diabetes

Hemiplegia

Moderate or severe renal disease

Diabetes with end-organ damage

\section{ICD-10 codes}

M05.0-M05.9, M06.0-M06.9, M08.0-M08.9, M13.0-M13.9, M15.0-M19.9, M47.0-M47.9, M48.0, M48.9

M80.0-M80.9, M81.0-M81.9, M82.0-M82.9, M83.0-M83.9

J45.0-J45.9

J43.0-J44.9

$120.0-120.9$

I05.0-107.9, I10.0-111.9, I13.0-I15.9, I24.0-124.9, I25.0-125.1, I25.3-125.9, I27.0-I27.9, I31.0-131.9, I34.0-135.9, |42.0-142.9, |44.0-146.9, |47.0-151.9

$121.0-121.9,122.0-122.9,125.2$

G00.0-G99.9

160.0-164.9, G45.0-G45.9

E10.0-E14.9

$173.9,170.2$

K20.0-K22.9, K25.0-K31.9

F32.0-F32.9, F33.0-F33.9

F40.0-F40.9, F41.0-F41.9

H53.0-H54.9

$\mathrm{H} 90.0-\mathrm{H} 91.9$

M50.0-M51.9

E66.0-E66.9

J45.0-J45.9, J43.0-J44.9

I05.0-107.9, I10.0-I11.9, I13.0-115.9, I24.0-124.9, I25.0-125.1, I25.3-125.9, I27.0-I27.9, I31.0-131.9, I34.0-135.9, |42.0-142.9, |44.0-146.9, |47.0-151.9, I20.0-120.9, I21.0-121.9, I22.0-122.9, I25.2

E10.0-E14.9

M48.0, M51.0, M51.1, M51.3-M51.9, M54.3-M54.6, M54.9

M13.0-M13.9, M15.0-M19.9, M47.0-M47.9, M48.0, M48.9

M05.0-M05.9, M06.0-M06.9, M08.0-M08.9

A00.0-B99.9, C00.0-C97.9, D00.0-D48.9, D50.0-D58.9, E00.0-E07.0, E15.0-E90.9, F00.0-F99.9, G00.0-G99.9, H00.0-H59.9, H60.0-H95.9, 100.0-199.9, J00.0-J39.9, J40.0-J42.9, J46.0-J99.9, K00.0-K93.9, L00.0-L99.9, M00.0-M03.9, M07.0-M07.9, M09.0-M12.9, M14.0-M14.9, M20.0-M46.9, M48.1-M48.8, M49.0-M99.9, N00.0-N99.9, O00.0-099.9, P00.0-P96.9, Q00.0-Q99.9, R00.0-R99.9, S00.0-S99.9, T00.0-T98.9

$121-124,125.2,125.8$

109, I11, 148, 149.0, 149.8, 150.0, 150.1, 150.1 + J81, 150.9, 151.5, 197.1

$170,171.1-171.6,171.8-171.9,172,173,174,177$

F00-F04, F05.1, F10

160-163, 165-168, G45

J41-J47

M05-M06, M08-M09, M12-M13, M30-M36

K25-K28

K70.0-K70.3, K70.9, K73, K74, K75.2-K75.9, K76.0-K76.5, K76.8-K76.9, K77

E10, E10.1, E10.5-E10.9, E11, E11.1, E11.5-E11.9, E13, E13.1, E13.5-E13.9, E14, E14.1, E14.5-E14.9

G81.0-G81.1, G81.9, 163, 166-167

I12-113, N00-N05, N17-N19

E10.2-E10.4, E11.2-E11.4, E13.2-E13.4, E14.2-E14.4 
Table 1 ICD-10 to comorbidity indices map (Continued)

\begin{tabular}{ll}
\hline Any tumour & D00-D48 \\
Leukaemia & C91-C95 \\
Lymphoma & C81-C85 \\
Moderate or severe liver disease & K70.4, K71.1, K71.7, K72, K75.0-K75.1, K76.6-K76.7 \\
Metastatic solid tumour & C00-C26, C30-C34, C37-C41, C43-C58, C60-C80, \\
AIDS & C88, C90, C96 \\
B20-B24
\end{tabular}

\section{ICD-10 chapters}

Indicator variables were generated for the presence or absence of conditions in Chapters I to XVII, and Chapter XIX. A variable indicating the number of chapters represented was generated, an approach used by Cameron et al. [10].

\section{Six frequent chronic conditions described by Haagsma et al.} (2011)

Haagsma et al. investigated the impact of comorbidity on disability weight estimates in a sample of 2,295 injured patients in the Netherlands [7]. The six most common self-reported chronic diseases were: (i) chronic non-specific lung disease; (ii) heart disease; (iii) diabetes; (iv) backache; (v) osteoarthritis; and (vi) rheumatoid arthritis. All other comorbidities are considered as "other". Indicator variables for these conditions were mapped from the ICD-10 codes (Table 1).

The key differences between the comorbidity indices used relate to the number of conditions represented. The ICD chapter approach maps all available ICD-10 comorbidity codes into 18 chapter-related groups, but does not specifically identify individual conditions. For example, diabetes is included in the "Endocrine, nutritional and metabolic disorders" chapter. The six frequent chronic conditions described by Haagsma et al. [7] also uses all available ICD-10 comorbidity codes but only six specific diagnoses are included, with all remaining comorbidities grouped together in an "other" category. The FCI includes only 18 conditions, with patients' ICD10 coded comorbidities not included in this list considered as having no comorbidities. The ICD-10 chapter, six frequent chronic conditions and FCI do not weight the severity of comorbidities. The CCI includes 19 conditions, but weights the severity of these conditions. For example, the presence of diabetes is given either a weighting of 1 or 2 depending on whether there is end-organ disease, and mild liver disease is differentiated from moderate/severe liver disease by CCI weightings.

The relationship between the conditions included in each comorbidity measure is complex. The ICD-10 chapter approach includes all conditions specified by the six frequent chronic condition, FCI and CCI comorbidity measures. All remaining measures include specific categories for heart disease, chronic pulmonary disease, and diabetes, although how this is represented varies (Table 1). For example, the six frequent common conditions approach bundles all diagnoses related to heart disease into a single category, while the FCI separates heart disease into three categories; angina, congestive heart failure (CHF)/heart disease, and heart attack. The CCI uses two categories for heart disease; myocardial infarction, and CHF. Arthritis is common to the six frequent chronic conditions and FCI approach, although the six frequent chronic conditions approach separates rheumatoid arthritis and osteoarthritis into individual categories. Similarly, the six frequent common conditions approach includes a category for "backache" while the FCI includes only a subset of relevant ICD-10 codes in its degenerative disc disease category (Table 1). Both the FCI and CCI include a specific category for peripheral vascular disease. Gastrointestinal and neurological disease are included in both the FCI and CCI but the specificity of categories differs between the two measures (Table 1). Only the FCI includes osteoporosis, obesity, depression, anxiety disorders, vision impairment and hearing impairment as individual categories, while only the CCI includes connective tissue disease, dementia, renal disease, metastatic disease, AIDS, leukemia, lymphoma, and other tumours as specific categories. Overall, the common groups of conditions represented in all comorbidity measures are diabetes, heart disease, and chronic pulmonary disease.

\section{Outcome measures}

Survivors to discharge in VOTOR are followed-up by standardised telephone interview at 12-months post-injury to collect disability outcomes [18]. The outcome of interest for this study was the Glasgow Outcome Scale Extended (GOS-E) which rates a person's function on an 8-level scale from 1 (death) to 8 (upper good recovery) [21]. The GOS-E is recommended for measurement of trauma patient outcomes [22,23], has demonstrated high levels of responsiveness to change in patients without head injury [24], and considers the patient's pre-injury function in the scoring process [21]. The GOS-E was dichotomised with a score $<7$ representing ongoing disability and 7-8 "recovery". 


\section{Data analysis}

A split dataset approach was used with the dataset randomly divided into two equal parts [25]. Models were developed using the "training" dataset and internally validated using the "test" dataset. Means and standard deviations (SD), or medians and interquartile range (IQR), were used to summarise continuous variables. Categorical variables were summarised using counts and percentages. Logistic regression models were fitted with "recovery" as the outcome. Model performance was measured using calibration and discrimination statistics. Age was categorised into eight groups, as age in its continuous form was not linearly related to the log odds of recovery.

Calibration measures how well the models predict over the entire range and was assessed using the HosmerLemeshow $(\mathrm{H}-\mathrm{L})$ statistic and calibration curves. A higher $\mathrm{H}$-L statistic and significant $\mathrm{p}$-value correspond to poorer calibration [26]. Calibration curves plot the observed proportion of events against the predicted probabilities of events, with perfect agreement between observed and predicted probabilities forming a $45^{\circ}$ line ("line of best fit") [25].

The area under the receiver operating characteristic (ROC) curve (AUC) measures the capacity of the model to discriminate between those who do and do not experience the outcome of interest [25]. ROC plots sensitivity against 1-specificity over the range of probabilities. Discrimination is generally classified as Acceptable (AUC $0.7-<0.8$ ), Excellent (AUC $0.8-<0.9$ ) and Outstanding (AUC $\geq 0.9$ ) [26].

In the training dataset, a model was fitted with injury variables and age as independent variables, and "recovery" as the dependent variable. Each measure of comorbidity was added and the models were compared using likelihood ratio (LR) tests, AUC (95\% CI), and the H-L statistic. The following comorbidity adjustment methods were used.

i. CCI weight categorised as $0,1, \geq 2$

ii. Number of ICD-10 chapters represented categorised as $0,1, \geq 2$

iii. FCI score categorised as $0,1, \geq 2$

iv. Number of the six frequent chronic conditions described by Haagsma et al. [7] represented categorised as $0,1, \geq 2$

v. All indicator variables for ICD-10 chapters included vi. All FCI condition indicator variables included

vii. All of the six frequent chronic condition indicator variables included.

The number of conditions/weighted index was not linearly related to the log odds of recovery, requiring categorisation. The individual conditions of the CCI were not modeled as the weighted index is the most commonly used form of the index. Models were then fitted to the test dataset and fit assessed using the AUC, H-L statistics, and calibration curves. Data were complete for all data items included in the models, ensuring that comparison between models was based on the same cases. All analyses were performed using Stata Version 11.0 (Stata Corp, College Station, Texas). A p-value $<0.05$ was considered significant.

\section{Results}

\section{Overview of the dataset}

There were 15,471 survivors to discharge, and 13,519 (87.4\%) had a valid GOSE-E at 12-months. Cases lost to follow-up at 12-months included a higher proportion of patients less than 45 years of age, male, and injured in motor vehicle crashes (Table 2). The overall distribution of orthopaedic injuries sustained was comparable between the groups, but the proportion without documented comorbidities able to be coded to the indices of interest was higher in the cases lost to follow-up (Table 2).

Most cases followed-up at 12-months post-injury had no comorbidity recorded in the ICD-10-AM diagnoses (Table 3). Mental and behavioural disorders (14\%), diseases of the circulatory system (10\%), and endocrine, nutritional and metabolic disorders (7\%) were most prevalent when using the ICD-10 chapters to classify comorbidity. Using the FCI, diabetes (6\%), heart disease (5\%) and neurological disease (5\%) were most prevalent, while "other" conditions (34\%) were most common using the six frequent chronic conditions described by Haagsma et al. (Table 3).

The random split resulted in 6,798 cases in the training dataset and 6,792 cases in the test dataset. Cases in the two datasets were comparable. The percentage of cases who had "recovered" by 12-months post-injury was $42 \%$ in both datasets.

\section{Contribution of age and comorbid conditions to prediction of 12-month disability \\ Training dataset}

Adding age resulted in improved model fit over adjustment for injuries alone (Table 4). The addition of comorbid status, irrespective of method of comorbid measurement, improved model fit further (Table 4). All comorbidity adjustment methods resulted in acceptable calibration (as tested using the H-L statistic), but use of the ICD-10 chapters and the six frequent chronic conditions approaches demonstrated higher AUC than adjustment using the FCI or CCI (Table 4). Adjustment for the number of comorbid conditions compared to adjusting for the presence or absence of each condition/chapter did not result in improved discrimination for the ICD-10 chapters $\left(\mathrm{X}_{1}^{2}=0.11, \mathrm{p}=0.743\right)$, FCI $\left(\mathrm{X}_{1}^{2}=0.37, \mathrm{p}=0.544\right)$ or the six frequent chronic conditions $\left(\mathrm{X}_{1}^{2}=1.21, \mathrm{p}=0.271\right)$. The 
Table 2 Comparison of orthopaedic trauma patients followed-up at 12-months and patients lost to follow-up

\begin{tabular}{|c|c|c|c|}
\hline \multicolumn{2}{|c|}{ Population characteristic } & \multirow{2}{*}{$\begin{array}{c}\begin{array}{c}\text { Followed-up } \\
(\mathbf{n}=\mathbf{1 3}, \mathbf{5 1 9})\end{array} \\
1704(12.6)\end{array}$} & \multirow{2}{*}{$\begin{array}{c}\begin{array}{c}\text { Lost to follow-up } \\
(\mathbf{n}=\mathbf{1}, \mathbf{9 5 2})\end{array} \\
356(18.2)\end{array}$} \\
\hline Age group & $15-24$ years & & \\
\hline & 25-34 years & $1672(12.4)$ & $404(20.7)$ \\
\hline & $35-44$ years & $1694(12.5)$ & $301(15.4)$ \\
\hline & $45-54$ years & $1655(12.2)$ & $220(11.3)$ \\
\hline & $55-64$ years & $1577(11.7)$ & $199(10.2)$ \\
\hline & $65-74$ years & $1480(10.9)$ & $157(8.0)$ \\
\hline & $75-84$ years & $2071(15.3)$ & $200(10.3)$ \\
\hline & $85+$ years & $1666(12.3)$ & $115(5.9)$ \\
\hline \multirow[t]{2}{*}{ Gender } & Male & $7361(54.5)$ & $1204(61.7)$ \\
\hline & Female & $6158(45.5)$ & $748(38.3)$ \\
\hline \multirow{8}{*}{$\begin{array}{l}\text { Mechanism } \\
\text { of injury }\end{array}$} & Low fall (<1 m) & $5390(39.9)$ & $613(31.4)$ \\
\hline & Motor vehicle & $1869(13.8)$ & $339(17.4)$ \\
\hline & High fall & $1816(13.4)$ & $254(13.0)$ \\
\hline & Motorcycle & $1349(10.0)$ & $208(10.7)$ \\
\hline & Pedestrian & $579(4.3)$ & $122(6.3)$ \\
\hline & Pedal cyclist & $616(4.6)$ & $71(3.6)$ \\
\hline & $\begin{array}{l}\text { Collision with object } \\
\text { or person }\end{array}$ & $505(3.7)$ & $120(6.1)$ \\
\hline & Other & $1395(10.3)$ & $225(11.5)$ \\
\hline \multirow[t]{10}{*}{ Injury group } & $\begin{array}{l}\text { Isolated lower } \\
\text { extremity fracture }\end{array}$ & $5271(39.0)$ & $701(35.9)$ \\
\hline & $\begin{array}{l}\text { Isolated upper } \\
\text { extremity fracture }\end{array}$ & $2809(20.8)$ & $478(24.5)$ \\
\hline & Spinal fractures only & $1741(12.9)$ & $254(13.0)$ \\
\hline & $\begin{array}{l}\text { Multiple lower } \\
\text { extremity fractures }\end{array}$ & $972(7.2)$ & $123(6.3)$ \\
\hline & $\begin{array}{l}\text { Upper and lower } \\
\text { extremity fractures }\end{array}$ & $620(4.6)$ & $87(4.5)$ \\
\hline & Soft tissue injury & $540(4.0)$ & $94(4.8)$ \\
\hline & $\begin{array}{l}\text { Spine and lower } \\
\text { extremity fractures }\end{array}$ & $465(3.4)$ & $64(3.3)$ \\
\hline & $\begin{array}{l}\text { Multiple upper } \\
\text { extremity fractures }\end{array}$ & $440(3.3)$ & $69(3.5)$ \\
\hline & $\begin{array}{l}\text { Spine and upper } \\
\text { extremity fracture }\end{array}$ & $431(3.2)$ & $50(2.6)$ \\
\hline & $\begin{array}{l}\text { Spine, upper and } \\
\text { lower extremity } \\
\text { fractures }\end{array}$ & $230(1.7)$ & $32(1.6)$ \\
\hline \multirow[t]{3}{*}{$\mathrm{CCl}^{\mathrm{a}}$ weight } & 0 & $9801(72.5)$ & $1489(76.3)$ \\
\hline & 1 & $2681(19.8)$ & $365(18.7)$ \\
\hline & $\geq 2$ & $1037(7.7)$ & $98(5.0)$ \\
\hline \multirow[t]{3}{*}{$\mathrm{FCl}^{\mathrm{b}}$ score } & 0 & 10859 (80.3) & $1675(85.8)$ \\
\hline & 1 & $1951(14.4)$ & $212(10.9)$ \\
\hline & $\geq 2$ & 709 (5.2) & $65(3.3)$ \\
\hline
\end{tabular}

Table 2 Comparison of orthopaedic trauma patients followed-up at 12-months and patients lost to follow-up (Continued)

\begin{tabular}{llcc}
\hline ICD-10 & 0 & $3515(26.0)$ & $554(28.4)$ \\
chapters & 1 & $2949(21.8)$ & $441(22.6)$ \\
& $\geq 2$ & $7055(52.2)$ & $957(49.0)$ \\
Haagsma & 0 & $8336(61.7)$ & $1259(64.5)$ \\
conditions [7] & 1 & $3979(29.4)$ & $594(30.4)$ \\
& $\geq 2$ & $1204(8.9)$ & $99(5.1)$ \\
\hline
\end{tabular}

${ }^{\mathrm{a}} \mathrm{CCl}$, Charlson Comorbidity Index; ${ }^{\mathrm{b}} \mathrm{FCl}$, Functional Comorbidity Index; ${ }^{\mathrm{C} C \mathrm{CD}}-10$, International Classification of Diseases, 10th Revision.

AUC was higher for the number of ICD-10 chapters represented when compared to the number of the six frequent chronic conditions represented $\left(X_{1}^{2}=8.75\right.$, $\mathrm{p}=0.003)$. However, the overall range of the AUC for the models adjusting for comorbidity ranged from 0.716 to 0.729 (Table 4).

\section{Test dataset}

Fitting the models to the test dataset resulted in a similar pattern of results but poorer model fit with none of the models demonstrating acceptable calibration and lower AUC than the training dataset models (Table 5). The AUC ranged from 0.691 to 0.704 with the number of ICD-10 chapters represented demonstrating the highest discrimination (Table 5). Despite poor calibration of models as measured using the H-L statistic, calibration curves tracked close to the line of best fit with the ICD10 and six frequent common condition curves showing better calibration at lower prediction percentiles and all models over-estimating recovery at higher prediction percentiles (Figure 1).

\section{Discussion}

The potential for comorbidity to impact on the long term disability experienced by injury survivors is clear but how best to adjust for comorbidity has not been well explored. This study of 13,519 injury survivors found that comorbidity, mapped to commonly used indices from routinely collected ICD-10 diagnosis codes, is an important predictor of functional recovery, providing additional predictive value over adjustment for age alone.

The findings confirm that comorbidity impairs patient recovery after injury. However, measurement of comorbidity remains a challenge. A lack of defined criteria for what constitutes a comorbidity, and absence of an established gold standard for measuring comorbidity [3,9], have led to a variety of methods and indices being used in injury research. Routinely collected discharge data, patient self-report and medical record review are common sources of comorbidity information [27,28]. Each source has strengths and weaknesses with self-report 
Table 3 Distribution of comorbid conditions in the dataset $(n=13,519)$

\begin{tabular}{|c|c|c|c|}
\hline \multicolumn{4}{|c|}{ Comorbidity measure } \\
\hline ICD-10 ${ }^{\text {a }}$ Chapters & n (\%) & $\mathrm{FCl}^{\mathrm{b}}$ conditions & n (\%) \\
\hline I - Infectious and parasitic diseases & $470(3.5)$ & Arthritis & $145(1.1)$ \\
\hline II - Neoplasms & $178(1.3)$ & Osteoporosis & $296(2.2)$ \\
\hline III - Diseases of blood and blood-forming organs & $723(5.4)$ & Asthma & $25(0.2)$ \\
\hline IV - Endocrine, nutritional, metabolic disorders & $900(6.7)$ & COPD/ARDS & $225(1.7)$ \\
\hline V - Mental and behaviour disorders & $1950(14.4)$ & Angina & $23(0.2)$ \\
\hline $\mathrm{VI}$ - Diseases of the nervous system & $783(5.8)$ & Congestive heart failure/Heart disease & $726(5.4)$ \\
\hline VII- Diseases of the eye and adnexa & $288(2.1)$ & Heart attack & $73(0.5)$ \\
\hline VIII - Diseases of the ear and mastoid process & $130(1.0)$ & Neurological disease & $656(4.9)$ \\
\hline IX - Diseases of the circulatory system & $1349(10.0)$ & Stroke or Transient Ischaemic Attack & $37(0.3)$ \\
\hline$X$ - Disease of the respiratory system & $424(3.1)$ & Diabetes & $815(6.0)$ \\
\hline$X I$ - Diseases of the digestive system & $347(2.6)$ & Peripheral vascular disease & $56(0.4)$ \\
\hline XII - Diseases of the skin, subcutaneous tissue & $332(2.5)$ & Upper gastrointestinal disease & $62(0.5)$ \\
\hline XIII - Diseases of the musculoskeletal system & $806(6.0)$ & Depression & $22(0.2)$ \\
\hline XIV - Diseases of the genitourinary system & $543(4.0)$ & Anxiety/panic disorders & $80(0.6)$ \\
\hline XV - Pregnancy, childbirth and the puerperium & $3(0.02)$ & Visual impairment & $101(0.8)$ \\
\hline $\mathrm{XVI}$ - Conditions originating in perinatal period & $1(0.01)$ & Hearing impairment & $93(0.7)$ \\
\hline XVII - Congenital malformations & $52(0.4)$ & Degenerative disc disease & $21(0.2)$ \\
\hline XIX - Injury, poisoning, etc. & $83(0.6)$ & Obesity & $135(1.0)$ \\
\hline Six frequent chronic conditions [7] & n (\%) & Charlson Comorbidity Index & n (\%) \\
\hline Chronic lung disease & $250(1.9)$ & Myocardial infarction & $238(1.8)$ \\
\hline Heart disease & $782(5.8)$ & Congestive heart failure & $378(2.8)$ \\
\hline Diabetes & $815(6.0)$ & Peripheral vascular disease & $19(0.1)$ \\
\hline Backache & $49(0.4)$ & Dementia & $1898(14.0)$ \\
\hline Osteoarthritis & $129(1.0)$ & Cerebrovascular disease & $46(0.3)$ \\
\hline Rheumatoid arthritis & $17(0.1)$ & Chronic pulmonary disease & $277(2.0)$ \\
\hline \multirow[t]{13}{*}{ Other } & 4555 (33.7) & Connective tissue disease & $37(0.3)$ \\
\hline & & Ulcer disease & $19(0.1)$ \\
\hline & & Mild liver disease & $98(0.7)$ \\
\hline & & Diabetes & $524(3.9)$ \\
\hline & & Hemiplegia & $155(1.1)$ \\
\hline & & Moderate or severe renal disease & $547(4.0)$ \\
\hline & & Diabetes with end-organ damage & $379(2.8)$ \\
\hline & & Any tumour & $65(0.5)$ \\
\hline & & Leukaemia & $13(0.1)$ \\
\hline & & Lymphoma & $2(0.01)$ \\
\hline & & Moderate or severe liver disease & $30(0.2)$ \\
\hline & & Metastatic solid tumour & $105(0.8)$ \\
\hline & & AIDS & $16(0.1)$ \\
\hline
\end{tabular}

${ }^{\mathrm{a}} \mathrm{CCD}-10$, International Classification of Diseases, 10th Revision; ${ }^{\mathrm{b}} \mathrm{FCl}$, Functional Comorbidity Index; ${ }^{\mathrm{C} C O P D}$, Chronic Obstructive Pulmonary Disease, ARDS, Adult Respiratory Distress Syndrome. 
Table 4 Discrimination and calibration of models in training dataset $(n=6798$ )

\begin{tabular}{lllll}
\hline Model & Comorbidity measure & $\begin{array}{c}\text { Area under curve } \\
(\mathbf{9 5 \%} \text { Cl) }\end{array}$ & $\begin{array}{c}\text { H-L statistic } \\
(\mathbf{p} \text {-value })\end{array}$ & $\begin{array}{c}\text { LR test } \\
(\mathbf{p} \text {-value })\end{array}$ \\
\hline Injury group & None & $0.631(0.618,0.644)$ & $0.67(0.954)$ & - \\
Injury group and age group & None & $0.704(0.692,0.716)$ & $4.04(0.854)$ & $507.40(<0.0001)$ \\
Injury group, age group and comorbidity & Number of ICD-10 chapters & $0.728(0.716,0.740)$ & $11.90(0.156)$ & $228.23(<0.0001)^{*}$ \\
Injury group, age group and comorbidity & ICD-10 chapters & $0.729(0.717,0.741)$ & $8.51(0.386)$ & $251.38(<0.0001)^{*}$ \\
Injury group, age group and comorbidity & Number of Haagsma conditions & $0.724(0.712,0.736)$ & $7.51(0.482)$ & $182.06(<0.0001)^{*}$ \\
Injury group, age group and comorbidity & Haagsma conditions & $0.725(0.713,0.737)$ & $7.05(0.531)$ & $194.03(<0.0001)^{*}$ \\
Injury group, age group and comorbidity & CCl weight category & $0.720(0.708,0.732)$ & $1.30(0.996)$ & $159.35(<0.0001)^{*}$ \\
Injury group, age group and comorbidity & $\mathrm{FCl} \mathrm{score}$ & $0.716(0.704,0.728)$ & $7.10(0.526)$ & $102.39(<0.0001)^{*}$ \\
Injury group, age group and comorbidity & $\mathrm{FCl} \mathrm{conditions}$ & $0.716(0.704,0.728)$ & $8.05(0.429)$ & - \\
\hline
\end{tabular}

${ }^{*}$ compared to model including age group and injury group.

criticised due to the potential for recall bias, difficulty in data collection with cognitive deficits, and prohibitive costs in large studies [28]. Medical record review is considered the most comprehensive method, with documented conditions corresponding well to established comorbidity indices, but is resource intensive [28]. In the current study, routinely collected discharge data were used to map ICD-10 diagnoses to comorbidity indices. Routinely collected discharge data have been described as "inevitably imperfect" [28] due to the coding of only a subset of recorded conditions and a focus on coding for maximising reimbursement in many settings. However, routinely collected discharge data provide a less resource intensive method for capturing comorbidities and have been shown to agree with medical record review for key conditions such as diabetes, cancer, chronic lung disease and alcohol abuse [29]. Studies directly comparing various data sources in trauma are absent, but Fan et al., in their study of $>10,000$ Veterans Affairs patients, found comparable prediction of health-related quality of life when comparing routinely collected discharge data with record review and self-report [27].

Our findings are consistent with previous studies that have found comorbidity based on patient or proxy self- report $[7,12,13,15,16]$ and registry data, [11] to be an important predictor of longer term functional or healthrelated quality of life outcome following injury. In contrast to previous studies, comorbidity provided additional predictive value over adjustment only for age [3-5]. Adjustment for the specific conditions showed little improvement over adjustment based on the number of conditions represented for the ICD-10 chapters, FCI and the six frequent chronic conditions reported by Haagsma et al., supporting methods of adjustment previously used $[10,11,15,16]$.

Discrimination and calibration statistics of the various models revealed relatively little difference in model prediction of functional recovery, with ICD-10 chapter and the six frequent chronic condition-based models demonstrating slightly better performance than FCI and CCI models. A potential explanation for the improved performance of the ICD-10 and the six frequent chronic condition models is that they used all of the available ICD-10 codes, and therefore all of the comorbidity information available, while the FCI and CCI restricted the conditions included in the model to a specific subset. In data not shown, only diabetes, heart disease, stroke, neurological disease, PVD and visual impairment were significant predictors of recovery in

Table 5 Discrimination and calibration of models in test dataset $(n=6721)$

\begin{tabular}{|c|c|c|c|}
\hline Model & Comorbidity measure & Area under curve $(95 \% \mathrm{Cl})$ & H-L statistic (p-value) \\
\hline Injury group & None & $0.600(0.587,0.613)$ & $35.52(<0.0001)$ \\
\hline Injury group and age group & None & $0.678(0.665,0.690)$ & $25.64(0.001)$ \\
\hline Injury group, age group and comorbidity & Number of ICD-10 chapters & $0.704(0.692,0.717)$ & $19.12(0.014)$ \\
\hline Injury group, age group and comorbidity & ICD-10 chapters & $0.703(0.691,0.716)$ & $22.61(0.004)$ \\
\hline Injury group, age group and comorbidity & Number of Haagsma conditions & $0.701(0.689,0.713)$ & $20.31(0.009)$ \\
\hline Injury group, age group and comorbidity & Haagsma conditions & $0.701(0.688,0.713)$ & $18.47(0.018)$ \\
\hline Injury group, age group and comorbidity & CCl weight category & $0.696(0.683,0.708)$ & $19.78(0.011)$ \\
\hline Injury group, age group and comorbidity & $\mathrm{FCl}$ score & $0.694(0.681,0.706)$ & $16.71(0.033)$ \\
\hline Injury group, age group and comorbidity & $\mathrm{FCl}$ conditions & $0.691(0.678,0.703)$ & $18.84(0.016)$ \\
\hline
\end{tabular}




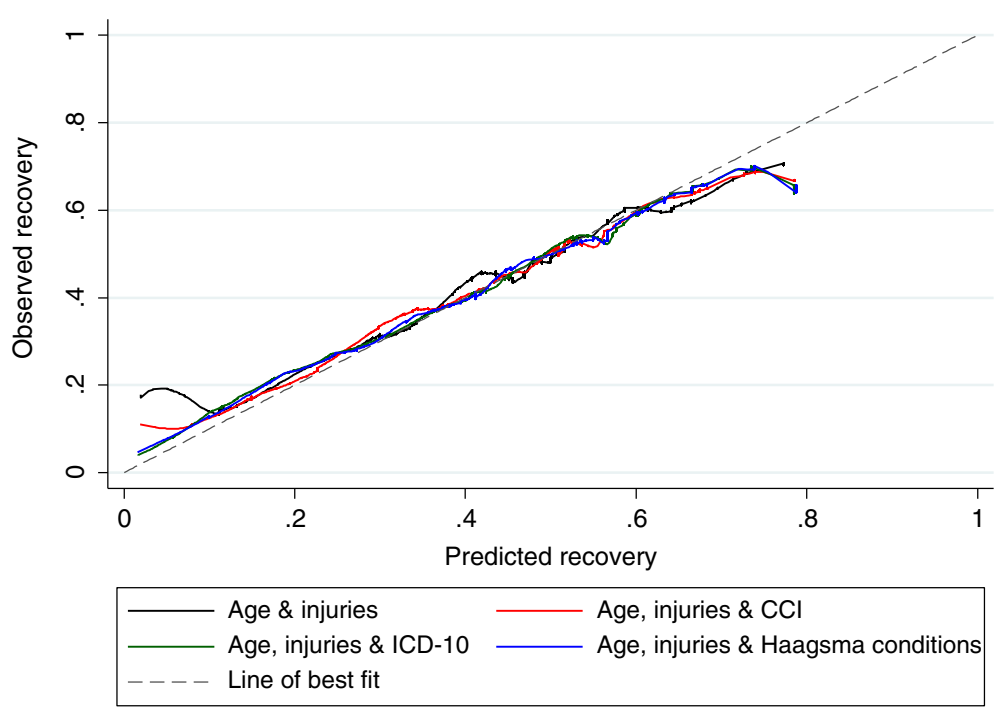

Figure 1 The figure is a plot the predicted versus the observed recovery in the test dataset. The $45^{\circ}$ line represents perfect fit of the model.

the FCI models. For the six frequent chronic condition models, diabetes, heart disease and "other" conditions remained significant. The ICD-10 chapters related to ear, respiratory, skin, musculoskeletal, digestive, genitourinary and congenital conditions failed to reach significance in the model.

The prevalence of osteoarthritis, rheumatoid arthritis and obesity in the study population was $1 \%, 0.1 \%$ and $1 \%$ (Table 2). The reported prevalence of these diseases in the Australian population is $15 \%,[30] 2 \%$,[31] and 19$22 \%$ [32], respectively. In contrast, the prevalence of heart disease in the study population was $6 \%$, slightly higher than the reported Australian population prevalence for heart, stroke and vascular conditions of $4 \%$ [33]. The prevalence of diabetes in the study population was $6 \%$, also slightly higher than the reported $4 \%$ national prevalence [34]. The differences in sample prevalence of diseases relative to population prevalence could be explained by the demographic profile of injury patients. In general, the trauma population tends to be younger and healthier than the general population. A number of prospective studies have shown pre-injury quality of life to be higher than age and gender matched population norms [35-37] and hence would be expected to have a lower prevalence of chronic diseases. However, the prevalence of some of these conditions (e.g. arthritis) is substantially lower than expected, particularly given the prevalence of elderly patients in the study sample and this is likely to reflect the ICD-10 coding practices and directives.

As noted in the methods, coders are directed to code conditions that have impacted on care provided during the patient's hospital stay, and this will underestimate the true prevalence of chronic conditions. The finding that the models performed similarly is more likely to reflect the types of conditions coded from the medical record and the importance of these conditions to patient care. All indices included conditions such as diabetes and heart disease which require ongoing clinical management during a patient's admission.

The overarching purpose of this study was to explore the relative contribution of each comorbidity measurement approach, and its contribution over age alone, to prediction of functional outcome. Importantly, despite models including age, injuries sustained and comorbidity, the capacity to discriminate between recovered/nonrecovered patients was only in the acceptable range (AUC $\approx 0.70$ ), confirming the importance of other factors. Numerous injury, personal and environmental factors have the capacity to influence the recovery of an individual. This is well demonstrated in the literature where factors such as compensable status [10,11,15,16,38,39], level of education $[11,13,16]$, the intent of injury [11], gender $[11,13,16,40,41]$, social circumstances $[13,16,18]$, intent [11], mechanism of injury [11], and the level of designation of the trauma centre of management $[11,42]$ have been shown to be important. While adjustment for comorbidity is important, the contribution of other factors cannot be under-estimated and should be part of any riskadjustment processes.

The strengths of this study are the large number of cases $(>13,000)$, high follow-up rate at 12 -months $(87 \%$ of all survivors to hospital discharge), and the use of a hospital discharge data to identify comorbidities which has been shown to have low error rates in diagnoses audited [43]. However, there were study limitations. 
The study focused on orthopaedic trauma cases rather than all admitted trauma cases. While many cases had also sustained non-orthopaedic injuries, the results may not reflect cases without orthopaedic injury and this should be considered when interpreting the findings. Nevertheless, the orthopaedic registry was selected as the data source specifically for this study because this population includes a higher proportion of elderly patients with comorbidities than other injury populations such as major trauma. There were differences between included cases and those lost to follow-up at 12-months with a bias towards older patients with comorbidity in the group followed-up and included in this study. Secondly, there are challenges in collecting comorbidity information from patients from large populations and particularly where cognitive deficit (e.g. head injury and pre-existing dementia) are prevalent. Therefore, we were not able to assess the agreement between self-report and ICD-10 coding, or fully evaluate the relationship between the FCI and the six frequent chronic conditions not prevalent in the ICD-10 codes, highlighting issues with adapting self-report based indices (e.g. the six frequent chronic conditions described by Haagsma et al.) to ICD-10 based datasets. In future, the development of privacy protecting record linkage systems may enable primary care data to be linked to trauma and hospital discharge datasets to permit evaluation of the impact of conditions not deemed to influence in-hospital care.

Each of the participating hospitals' coders used the same coding standard but the potential for variable interpretation of the medical record and the coding directives remains, despite regular auditing of the hospital discharge data. Additionally, ICD-10-AM coding is done in Australia for reimbursement purposes. In other jurisdictions, where ICD-10 coding is not used for reimbursement or where systems limit the number of codes recorded, the number and distribution of codes may differ. Whether this would impact on ICD-10 based comorbidity adjustment is unknown but warrants consideration in the interpretation of the findings and for further research. Finally, this study focused on functional outcome. The possibility that the relationship between comorbidity and other outcomes such as health-related quality of life, return to work and pain differs from the relationship between comorbidity and functional status will be examined in further research using data from VOTOR.

\section{Conclusions}

Mapping of ICD-10 codes to comorbidity indices showed that comorbidity is an important predictor of long term functional outcome following orthopaedic trauma, independent of age and injuries sustained.
Adjustment for comorbidity is indicated when assessing risk-adjusted functional outcomes over time or across jurisdictions.

\section{Competing interests}

The authors declare that they have no competing interests.

\section{Authors' contributions}

All authors contributed to the conception and design and interpretation of data. BJG analysed the data for this study. BJG drafted the article and all authors reviewed it critically for important intellectual content. All authors approved the final version for submission.

\section{Acknowledgements}

The Victorian Orthopaedic Trauma Outcomes Registry (VOTOR) is funded by the TAC through the Institute for Safety, Compensation and Recovery Research. Associate Professor Belinda Gabbe and Professor Peter Cameron were supported by a Career Development Award, and a Practitioner Fellowship, from the National Health and Medical Research Council of Australia, respectively. The authors would like to acknowledge the staff, participating hospitals and Steering Committee of VOTOR.

\section{Author details}

Department of Epidemiology and Preventive Medicine, Monash University, The Alfred Centre, 99 Commercial Rd, Melbourne, Victoria 3004, Australia. ${ }^{2}$ National Trauma Research Institute, The Alfred Hospital, Melbourne, Australia. ${ }^{3}$ Research Centre for Injury Studies, Flinders University, Adelaide, Australia. ${ }^{4}$ College of Medicine, Swansea University, Swansea, United Kingdom. ${ }^{5}$ Department of Orthopaedic Surgery, The Alfred, Melbourne, Australia. ${ }^{6}$ Emergency and Trauma Centre, The Alfred Hospital, Melbourne, Australia.

Received: 8 June 2012 Accepted: 23 January 2013 Published: 26 January 2013

\section{References}

1. Morris J Jr, MacKenzie E, Edelstein S: The effect of preexisting conditions on mortality in trauma patients. J Am Med Assoc 1990, 263(14):1942-1946.

2. Tan C, Ng A, Civil I: Co-morbidities in trauma patients: common and significant. N Z Med J 2004, 117(1201):1044-1049.

3. Wardle T: Co-morbid factors in trauma patients. Brit Med Bull 1999, 55(4):744-756.

4. Bergeron E, Rossignol M, Osler T, Clas D, Lavoie A: improving the TRISS methodology by restructuring age categories and adding comorbidities. J Trauma 2004, 56(4):760-767.

5. Gabbe B, Magtengaard K, Hannaford A, Cameron P: Is the Charlson Comorbidity Index useful for predicting trauma outcomes? Acad Emerg Med 2005, 12:318-321.

6. McGwin G, MacLennan P, Bailey Fife J, Davis G, Rue L: Preexisting conditions and mortality in older trauma patients. J Trauma 2004, 56(6):1291-1296.

7. Haagsma J, van Beeck E, Polinder S, Toet H, Panneman M, Bonsel G: The effect of comorbidity on health-related quality of life for injury patients in the first year following injury: comparison of three comorbidity adjustment approaches. Pop Health Metr 2011, 9:10.

8. Polinder S, Haagsma J, Lyons R, Gabbe B, Ameratunga S, Cryer C, Derrett S, Harrison J, Segui-Gomez M, van Beeck E: Measuring the population burden of fatal and non-fatal injury. Epidemiol Rev 2012, 34:17-31.

9. de Groot V, Beckerman H, Lankhorst G, Bouter L: How to measure comorbidity: a critical review of available methods. J Clin Epidemio/ 2003, 56(3):221-229.

10. Cameron C, Purdie D, Kliewer E, McClure R: Differences in prevalence of pre-existing morbidity between injured and non-injured populations. Bull World Health Org 2005, 83(5):345-352.

11. Gabbe B, Simpson P, Sutherland A, Wolfe R, Fitzgerald M, Judson R, Cameron P: Improved functional outcomes for major trauma patients in a regionalised, inclusive trauma system. Ann Surg 2012, 255(6):1009-1015.

12. Haentjens $P$, Autier $P$, Barette $M$, Venken $K$, Vanderschueren $D$, Boonen $S$ : Survival and functional outcome according to hip fracture type: A oneyear prospective cohort study in elderly women with an intertrochanteric or femoral neck fracture. Bone 2007, 41:958-964. 
13. Holtslag $H$, Van Beeck E, Lindeman E, Leenen L: Determinants of long-term functional consequences after major trauma. J Trauma 2007, 62(4):919-927.

14. Kelley-Quon L, Min L, Morley E, Hiatt J, Cryer H, Tillou A: Functional status after injury: A longitudinal study of geriatric trauma. Amer Surg 2010, 76(10):1055-1058.

15. Polinder S, Haagsma J, Bonsel G, Essink-Bot $M$, Toet $H$, van Beeck E: The measurement of long-term health-related quality of life after injury: comparison of EQ-5D and the health utilities index. Inj Prev 2010, 16:147-153.

16. Ringburg A, Polinder S, van lerland M, Steyerberg E, van Lieshout E, Patka P, van Beeck E, Schipper I: Prevalence and prognostic factors of disability after major trauma. J Trauma 2011, 70(4):916-922.

17. Edwards E, Graves S, McNeil J, Williamson O, Urquhart D, Cicuttini F: Orthopaedic trauma: Establishment of an outcomes registry to evaluate and monitor treatment effectiveness. Injury 2006, 37:95-96.

18. Gabbe B, Sutherland A, Hart M, Cameron P: Population-based capture of long-term functional and quality of life outcomes after major trauma the experiences of the Victorian State Trauma Registry. J Trauma 2010, 69(3):532-536

19. National Centre for Classification in Health: International Statistical Classification of Diseases and Related Health Problems, Tenth Revision, Australian Modification (ICD-10-AM), the Australian Classification of Health Interventions (ACHI) and the Australian Coding Standards (ACS). 7th edition. Sydney: 2010. ISBN 9781742101583

20. Groll D, To T, Bombardier C, Wright J: The development of a comorbidity index with physical function as the outcome. J Clin Epidemiol 2005, 58:595-602.

21. Wilson J, Pettigrew L, Teasdale G: Structured interviews for the Glasgow Outcome Scale and the Extended Glasgow Outcome Scale: Guidlines for their use. J Neurotrauma 1998, 15(8):573-585.

22. Gabbe B, Williamson O, Cameron P, Dowrick A: Choosing outcome assessment instruments for trauma registries. Acad Emerg Med 2005, 12:751-758.

23. Ardolino A, Sleat $G$, Willett $\mathrm{K}$ : Outcome measurements in major trauma Results of a consensus meeting. Injury 2012, 43:1662-1666.

24. Williamson O, Gabbe B, Forbes A, Wolfe R, Sutherland A, Cameron P: Comparing the responsiveness of functional outcome assessment instruments for trauma registries. J Trauma 2011, 71(1):63-68.

25. Altman D, Vergouwe $Y$, Royston P, Moons K: Prognosis and prognostic research: validating a prognostic model. Brit Med J 2009, 338(b605):1432-1435.

26. Hosmer D, Lemeshow S: Applied Logistic Regression, Second Edition edn. New York: John Wiley \& Sons, Inc:; 2000.

27. Fan V, Maciejewski M, Liu C, McDonell M, Fihn S: Comparison of risk adjustment measures based on self-report, administrative data, and pharmacy records to predict clinical outcomes. Health Serv Outcomes Res Method 2006, 6:21-36.

28. Lash T, Mor V, Wieland D, Ferrucci L, Satariano W, Silliman R: Methodology, design, and analytic techniques to address measurement of comorbid disease. J Gerontol A Biol Sci Med Sci 2007, 62(3):281-285.

29. Chong W, Ding Y, Heng B: A comparison of comorbidities obtained from hospital administrative data and medical charts in older patients with pneumonia. BMC Heal Serv Res 2011, 11:105.

30. March L, Bagga H: Epidemiology of osteoarthritis in Australia. Med J Aust 2004, 180(5 Suppl):S6-S10

31. Australian Institute of Health and Welfare: A snapshot of arthritis in Australia 2010, Arthritis series no 13. Canberra: Australian Institute of Health and Welfare; 2010. http://www.aihw.gov.au/publication-detail/? id $=6442468397 \& \mathrm{tab}=2$

32. Thorburn A: Prevalence of obesity in Australia. Obes Rev 2005, 6(3):187-189.

33. Australian Institute of Health and Welfare: Cardiovascular disease: Australian facts 2011, Cardiovascular disease series. Canberra: Australian Institute of Health and Welfare; 2011. http://www.aihw.gov.au/publication-detail/? id $=10737418510 \& \mathrm{tab}=2$.

34. Australian Institute of Health and Welfare: Diabetes prevalence in Australia, Diabetes Series. Canberra: Australian Institute of Health and Welfare; 2009. http://www.aihw.gov.au/publication-detail/?id=6442468288\&tab=2.

35. Gabbe B, Cameron P, Graves S, Edwards E: Pre-injury status: Are orthopaedic trauma patients different to the general population? J Orthop Trauma 2007, 21(4):223-228.

36. Lyons R, Kendrick D, Towner E, Christie N, Macey S, Gabbe B: Measuring the population burden of injuries - Implications for global and national estimates: A multi-centre prospective UK longitudinal study. PLOS Med 2011, 8(12):e1001140.

37. Watson W, Ozanne-Smith J, Richardson J: Retrospective baseline measurement of self-reported health status and health-related quality of life versus population norms in the evaluatin of post-injury losses. Inj Prev 2007, 13:45-50.

38. Gabbe B, Cameron P, Williamson O, Edwards E, Graves S, Richardson M: The relationship between compensable status and long-term patient outcomes following orthopaedic trauma. Med J Aust 2007, 187(1):14-17.

39. Harris I, Young J, Rae H, Jalaludin B, Solomon M: Predictors of general health after major trauma. J Trauma 2008, 64(4):969-974.

40. Holbrook T, Hoyt D, Anderson J: The importance of gender on outcomes after major trauma: functional and psychologic outcomes in women vesus men. J Trauma 2001, 50:270-273.

41. Vles W, Steyerberg E, Essink-Bot M, van Beeck E, Meeuwis J, Leenen L: Prevalence and determinants of disabilities and return to work after major trauma. J Trauma 2005, 58:126-135.

42. Mackenzie E, Rivara F, Jurkovich G, Nathens A, Egleston B, Salkever D, Frey $K$, Scharfstein D: The impact of trauma-center care on functional outcomes following major lower limb trauma. J Bone Joint Surg 2008, 90-A(1):101-109.

43. Henderson T, Shepheard J, Sundararajan V: Quality of diagnosis and procedure coding in ICD-10 administrative data. Med Care 2006, 44(11):1011-1019.

doi:10.1186/1472-6963-13-30

Cite this article as: Gabbe et al: Comparison of measures of comorbidity for predicting disability 12-months post-injury. BMC Health Services Research 2013 13:30.

\section{Submit your next manuscript to BioMed Central and take full advantage of:}

- Convenient online submission

- Thorough peer review

- No space constraints or color figure charges

- Immediate publication on acceptance

- Inclusion in PubMed, CAS, Scopus and Google Scholar

- Research which is freely available for redistribution 